\title{
Development of the Red Sequence in Galaxy Clusters
}

\author{
Noelia Jiménez ${ }^{1}$, Sofía A. Cora ${ }^{1}$, \\ Analía Smith Castelli ${ }^{1}$, and Lilia P. Bassino ${ }^{1}$ \\ ${ }^{1}$ Facultad de Ciencias Astronómicas y Geofísicas de La Universidad Nacional de La Plata and \\ Instituto de Astrofísica de La Plata (CCT La Plata, CONICET, UNLP), Argentina \\ Email: njimenez@f caglp.unlp.edu.ar
}

\begin{abstract}
We investigate the origin of the color-magnitude relation (CMR) observed in cluster galaxies by using a combination of a cosmological $N$-body simulation of a cluster of galaxies and a semi-analytic model of galaxy formation. The departure of galaxies in the bright end of the CMR with respect to the trend defined by less luminous galaxies could be explained by the influence of minor mergers.
\end{abstract}

Keywords. galaxies: clusters, galaxies: evolution

With the aim of understanding the development of the CMR depicted by earlytype galaxies in galaxy clusters, we apply a semi-analtytic model of galaxy formation (Lagos et al. 2008) to a simulated galaxy cluster with virial mass $\sim 1.3 \times 10^{15} h^{-1} M_{\odot}$ (Dolag et al. 2005). The semi-analtytic model considers gas cooling, star formation, galaxy mergers, disk instabilities, metal enrichment and feedback from supernovae, and active galactic nuclei. Photometric properties of simulated early-type galaxies are compared with those of early-type galaxies observed in the central region of the Antlia cluster (Smith Castelli et al. 2008); all magnitudes are obtained in the Washington photometric system $\left(C, T_{1}\right)$. We find that the general trend of simulated and observed CMRs are quite similar. However, the more massive simulated galaxies $\left(-22<M_{T_{1}}<-19 \mathrm{mag}\right)$ depart from the fit to observed data, displaying an almost constant color $\left(C-T_{1} \approx 1.7\right)$, as detected in other clusters. We select galaxies in six magnitude bins within the range $-22<M_{T_{1}}<-16 \mathrm{mag}$, and analyze the contribution to the stellar component of quiescent star formation and starbursts during disk instabilities and merger events. Major and minor mergers are distinguished between dry and wet mergers according to the cold gas mass of the remnant. For the most luminous galaxies, we find that the increase of stellar mass at low redshift arises as a consequence of minor dry merger events, in agreement with Skelton et al. (2009). Galaxies lying in the faint end of the CMR $\left(-17<M_{T_{1}}<-16\right.$ mag) mainly increase their stellar mass as a result of disk instabilities. This seems to indicate that the effect of minor dry mergers would be to increase the luminosity without strongly affecting the galaxy colors. We will deepen this investigation by focusing on the chemical enrichment history of the accreted stellar mass with the aim of disentangling the role played by dry mergers in determining the properties of the more massive galaxies.

\section{References}

Dolag, K., Vazza, F., Brunetti, G., \& Tormen, G. 2005, MNRAS, 364, 753

Lagos, C., Cora, S. A., \& Padilla, N. D. 2008, MNRAS, 388, 587

Skelton, R. E., Bell, E. F., \& Somerville, R. S. 2009, ApJ, 699, L9

Smith Castelli, A., et al. 2008, MNRAS, 386, 2311 